\title{
Application Exploration in Virtual Reality Integrated Learning Environment-Taking Digital Inquiry Laboratory as an Example
}

\author{
Wenjing Xia \\ Zhejiang Open University, Hangzhou, China \\ Email: 838814586@qq.com
}

How to cite this paper: Xia, W. J. (2022). Application Exploration in Virtual Reality Integrated Learning Environment-Taking Digital Inquiry Laboratory as an Example. Creative Education, 13, 75-85.

https://doi.org/10.4236/ce.2022.131005

Received: December 20, 2021

Accepted: January 15, 2022

Published: January 18, 2022

Copyright $\odot 2022$ by author(s) and Scientific Research Publishing Inc. This work is licensed under the Creative Commons Attribution International License (CC BY 4.0).

http://creativecommons.org/licenses/by/4.0/

\begin{abstract}
With the increasing popularity of virtual learning, the disadvantages of learners' operation ability and on-the-spot experience in the virtual learning environment are slightly inferior to those in the real learning environment. We "integrate" the "real" learning environment with the "virtual" learning environment based on network/multimedia to make it an organic whole and create a "virtual real" learning environment. Taking the digital inquiry laboratory as an example, this paper lists the characteristics of the new experimental method and its role in specific teaching under the support of virtual reality integration technology. With a view to reflecting the characteristics of learning activities in the virtual and real integration learning environment through examples.
\end{abstract}

\section{Keywords}

Virtual-Reality Learning Environment, Digital Inquiry Laboratory, Instructional Design

\section{Introduction}

Under the background of the rapid development of technology, represented by modern information technology such as multimedia, computer and network, more and more tools participate in the learning process, which makes more and more students build knowledge in the "virtual" learning environment. Compared with the limitations of traditional teaching activities in time and space, students' autonomy can be brought into full play in the "virtual" learning environment. Therefore, virtual reality has been widely sought after. However, we have to pay attention to the increasing popularity of virtual learning, the disadvantages of learners' operation ability and on-the-spot experience in the virtual 
learning environment are slightly inferior to those in the real learning environment. Considering the advantages and disadvantages of "virtual" and "real" learning environment, it seems that we should integrate the "real" learning environment inside/outside the classroom with the "virtual" learning environment based on network/multimedia, making it an organic whole and create a "virtual real" learning environment (Viitaharju et al., 2021).

Fortunately, with the support of sensor technology, today's virtual reality fusion learning environment is no longer a castle in the air. The virtual reality fusion teaching environment can meet the needs of learners for network collaborative learning while obtaining real learning experience (Qian \& Deng, 2006). In this paper, taking the digital inquiry laboratory as an example, the characteristics of the new experimental method and its role in specific teaching will be listed under the support of virtual reality integration technology. With a view to reflecting the characteristics of learning activities in the virtual and real integration learning environment through examples.

\section{Virtual Reality Integrated Learning Environment}

Nowadays, with the rapid development of social technology, especially represented by modern information technology such as multimedia, computer and network, these tools participate in the learning process, so that students can construct knowledge in a "virtual" learning environment. Compared with the limitations of traditional teaching activities in time and space, students' autonomy can be brought into full play in the "virtual" learning environment. Therefore, virtual reality has been widely sought after. However, we have to pay attention to that in the virtual learning environment, learners' operation ability and on-the-spot experience are slightly inferior to those learning in the real learning environment. Considering the advantages and disadvantages of "virtual" and "real" learning environment, it seems that we should find a way to integrate the "real" learning environment inside/outside the classroom with the "virtual" learning environment based on network/multimedia, so as to make it an organic whole and create a "virtual real" learning environment.

\subsection{Concept}

The so-called virtual real fusion learning environment refers to a new type of learning environment that identifies and obtains objective information related to learning activities in the real environment through sensor devices, and integrates the real learning environment based on classroom and society with the virtual learning environment based on network and multimedia through the Internet (Wang et al., 2020). In the virtual reality fusion learning environment, sensors play an indispensable role. The national standard gb7665-87 defines the sensor as "a device or device that can feel the specified measured parts and convert them into usable signals according to a certain law (mathematical function law), which is usually composed of sensitive elements and conversion elements". 
From this definition, it can be seen that as an important element in the virtual reality fusion learning environment, sensors can collect the effective information of learning activities in the real world and use it for learners through digital processing.

The application of Internet and multimedia technology in virtual reality integrated learning environment is mainly reflected in the construction of virtual learning environment and digital learning resources. The so-called virtual learning environment is to combine remote interaction and face-to-face interaction in a variety of ways, and provides a virtual space-time learning environment. Learners obtain relevant digital learning resources through the Internet and carry out learning inquiry activities in the virtual learning environment. In this way, learners' learning efficiency can be improved and cooperative learning activities can be carried out effectively.

\subsection{Research Status}

Many experts and scholars in the field of education have discussed how to effectively integrate computer technology into science curriculum in different dimensions and levels. Among them, most of them are "microcomputer based laboratory", "calculator based laboratory", "data acquisition system" and "handheld system" (Troutman \& Grimm, 2020). These studies have some commonalities, The main manifestations are as follows: 1) existing studies have focused on the application of digital technology in scientific topics and scientific inquiry activities; 2) studies have affirmed the positive impact of digital technology integration and science curriculum on teaching methods or teaching quality; 3) When the research emphasizes the problems of digital technology and the cultivation of students' inquiry ability to varying degrees, it rarely carries out in-depth discussion or gives specific suggestions and practices, and basically only stays in the discussion of interview records of teachers or students.

\subsection{Characteristics of Application in Virtual Reality Integrated Learning Environment}

\subsubsection{Accurate Information Transmission}

In the learning environment of virtual reality integration, due to the use of computer network, the collected data can be transmitted timely and accurately. The application of simultaneous interpreting and learning based on different learning environments ensures the scientificity of the sensor's use. The sensitivity of different sensors to different variables ensures that all applications designed in the virtual reality fusion teaching environment are targeted (Liu, Wang, \& Yin, 2018). When all sensors communicate with computers, various network protocols ensure the authenticity and accuracy of data information transmission.

\subsubsection{Timely Information Judgment}

In the virtual reality fusion learning environment, in order to meet the needs of different applications, there are a large number of sensors with different types 
and functions. These sensors provide a lot of information for the virtual reality fusion learning environment. The intervention of these sensors makes the information judgment process more rapid. Compared with manual information processing, the intelligent application makes the difficulties encountered in the manual processing stage, such as lack of attention, lack of experience and so on.

\subsubsection{Intelligent Information Processing}

As mentioned above, the biggest feature of virtual reality fusion learning environment is the participation of computer networks and sensors (Ma \& Li, 2012). In the virtual reality fusion learning environment, information acquisition is only a basic task. Through the cooperation of sensors and collectors and the participation of software, the information processing process will become intelligent. Based on all collected data, the intelligent system will process accordingly. In this way, the automatic intelligent control technology will involve various fields.

\section{Brief Introduction of Digital Inquiry Laboratory}

\subsection{Background of Digital Inquiry Laboratory}

At present, China is waking up the vigorous curriculum reform in primary and secondary schools. According to the requirements on the reform of teaching structure in the curriculum reform document, we should promote the integration of information technology and subject courses, gradually realize the changes in the presentation of teaching content, students' learning methods, teachers' teaching methods and teacher-student interaction methods, and give full play to the advantages of information technology, Provide a rich and colorful educational environment and powerful learning tools for students' learning and development (Li, 2019). The curriculum reform takes strengthening students' self-help inquiry learning as its core concept. Under this new situation, the status of the laboratory is particularly important. Much knowledge needs to be obtained through students' personal practice, which puts forward higher requirements for the laboratory.

In the past study, the traditional laboratory has exposed the following defects: first, the resolution of traditional experimental instruments is not high, which affects the results of inquiry experiments; Second, in the traditional laboratory, the experimental data can only be recorded manually, and the data sampling rate is not high, which has a great impact on the accuracy of the experimental results; Third, the ability of middle school students to analyze and process data is not strong. Under the influence of these adverse factors, in the long run, students will find the experiment difficult and of little significance, which seriously affects students' interest in learning.

In this situation, coupled with the rapid development of science and technology in the new era, digital inquiry laboratory came into being. Digital inquiry laboratory mainly uses computer technology, network communication technology, multimedia technology, etc. these technologies are mainly used to digitize 
various resources in the traditional laboratory, and realize the high efficiency of experimental teaching management through computer, so as to extend the laboratory in time and space (Hao et al., 2015).

In the process of exploring in the digital inquiry laboratory, students can, under the guidance of teachers, select and determine the theme of the inquiry experiment according to the teaching content and their respective interests, from curriculum learning, social phenomena, natural phenomena and self life, carry out experimental inquiry independently and cooperatively, actively acquire and apply knowledge and solve problems, so as to experience and understand the process of scientific exploration, develop the consciousness, quality and habit of independent exploration and innovation, and form and improve the creative ability (Wang, 2020). This process reflects the openness of digital inquiry laboratory in time, space and inquiry content. Digital inquiry laboratory is not only the digitization of experimental technology, but also the digitization of experimental management and operation. Digital laboratory is a student-centered, open and humanized laboratory (Pan, Luo, \& He, 2020). At the same time, in terms of laboratory management, digital inquiry laboratory is an efficient and programmed management system. In the exploration of this paper, mainly around the digital exploration process, the composition, characteristics and specific applications of digital laboratory will be listed.

\subsection{Composition of Digital Inquiry Laboratory}

As a new experimental teaching mode, digital inquiry laboratory broke through the ground under the background of the new curriculum reform of Science in middle schools in China. It is reported that China's Ministry of education cooperated with Tianjin No. 1 middle school to set up the country's first digital inquiry laboratory in 2004. As an inevitable product under the background of the development of the times, digital inquiry laboratory has long been used in the teaching process of physics, chemistry and biology in middle schools in Europe and America.

The digital inquiry laboratory under the background of the new curriculum is based on the real experiment, through various sensors to replace the traditional instruments, and directly hand over the collected accurate experimental data to the data processor through the interface equipment. After analysis and processing, it can more clearly and clearly show the phenomenon and reveal the scientific law. Digital inquiry laboratory seems to be a reform of traditional laboratory, but in fact it is an effective supplement to traditional laboratory teaching.

\subsubsection{Sensors}

Sensor (English Name: transducer/sensor) is a kind of detection device, which can feel the measured information and transform the sensed information into electrical signal or other required forms of information output according to a certain law, so as to meet the requirements of information transmission, processing, 
storage, display, recording and control. It is the primary link to realize automatic detection and automatic control (Jiang, Ni, \& Liu, 2021). (Baidu Encyclopedia) the national standard gb7665-87 defines a sensor as "a device or device that can feel the specified measured part and convert it into a usable signal according to a certain law (mathematical function law), which is usually composed of sensitive elements and conversion elements".

In the laboratory environment, compared with the five human sensory organs, the commonly used sensors include the following categories:

Hearing-sound sensitive sensor: such as sound intensity sensor and sound wave sensor.

Vision-photosensitive sensor: such as light door sensor and wireless photoelectric door sensor.

Smell-gas sensor: such as oxygen sensor and carbon dioxide sensor.

Taste-chemical sensor: such as electrolyte sensor and polymer sensor.

Tactile-pressure sensitive and temperature sensitive: such as pressure sensor and temperature sensor.

In the digital exploration laboratory, the role of the sensor is to transmit all kinds of non electrical signals measured in the experiment (for example, force, displacement, light intensity, $\mathrm{pH}$ value, etc.) are converted into standard electrical signals and transmitted to the collector. Through the use of sensors, the disadvantages of inaccurate data acquisition in traditional laboratories are effectively controlled. In the research of basic disciplines, sensors have a more prominent position. Whether it is observing the vast universe, observing the particle world, or even Deepen material understanding, developing new energy, new materials and other extreme technology research that plays an important role, such as ultra-high temperature, ultra-low temperature, ultra-high pressure, ultra-high vacuum, super magnetic field, ultra-weak magnetic field and so on. Through the use of sensors, these information that cannot be accurately obtained by experimenters or even by human senses will be accurately recorded for experiments. Therefore, on the one hand, the emergence of sensors increases the accuracy of experimental data, on the other hand, it is also conducive to the breakthrough of research in the subject field.

\subsubsection{Collector}

Data collector is an electronic instrument and an automatic equipment with the function of on-site real-time data acquisition and processing. In the digital exploration laboratory, the collector mainly plays the role of converting the analog signal of the sensor into a digital model and communicating with the computer. The data collector can record the changes of temperature, humidity, voltage and pressure of the surrounding environment for a period of time. Through the use of the collector, the authenticity, effectiveness, real-time and availability of field data are guaranteed in the digital inquiry laboratory. In the experiment, according to the different experimental types and experimental environment, an appropriate data collector is introduced to collect and transmit the effective data to 
the computer, which is convenient for unified data processing in the process of the experiment. According to the composition of existing data collectors, most of them have the characteristics of small volume, light weight and high performance. This provides a guarantee for students or teachers to provide handheld devices in the laboratory.

In addition to providing a new test method integrated with information technology, the data collector stimulates students' enthusiasm to participate in the experiment. At the same time, according to the objective advantages of precision and intelligence of the data collector, the use of the data collector in relevant experiments will undoubtedly reduce the troubles that will be encountered in the manual working diagram, manual counting and even in the data processing stage, It saves a lot of time for the data acquisition stage in the experimental process.

\subsubsection{Special Software for Digital Inquiry Laboratory}

In the digital inquiry laboratory, data is collected and expressed mainly through the division of labor and cooperation of sensors and collectors. According to the needs of specific experiments, supporting different special software for digital inquiry laboratory can analyze the collected data more efficiently. During the experiment, firstly, the sensor will measure various physical quantities and transmit the measured values or results to the data collector; Then the data collector transmits the collected experimental data to the computer; Finally, the experimental data are expressed in various ways such as numerical value, chart and curve through computer software, and the experimental data can be analyzed and processed according to the teaching requirements. In terms of data representation and analysis, these softwares can provide data tables, data curves and other forms. In the data analysis stage, a variety of analysis methods can be provided, such as linear regression, curve fitting, etc. In this way, the data recorded by students during the experiment can be processed more quickly, efficiently and accurately, and the experimental results can be verified faster. In the existing digital inquiry laboratory, the collector is connected with the computer through USB interface, which makes the data transmission process more stable and improves the compatibility at the same time.

\subsection{Characteristics of Digital Inquiry Laboratory System}

\subsubsection{Intelligence}

In the digital laboratory, the sensor is used as a tool to collect experimental data, and its resolution is much higher than that of traditional experimental instruments. From this point of view, it is decided to experiment in the digital laboratory, and the data accuracy and accuracy will be much higher than that of the traditional laboratory. In addition, because the data can be collected through the collector in the digital exploration laboratory, the data sampling rate will be more scientific and reasonable. At the same time, the higher sampling frequency also ensures the accuracy of the experimental results. Even through the interven- 
tion of the collector, many experiments involving continuous and rapid changes will be carried out in the laboratory. Finally, the software part of the intelligent digital exploration experimental platform also has powerful data processing function. Because in the process of building the laboratory, different laboratories will be equipped with the most appropriate special software for the three digital exploration laboratory. These softwares will help students quickly process the experimental results, facilitate students to efficiently complete scientific experiments and obtain experimental results. In this way, students will have more time for experimental reflection, and even reverification or discovery of scientific laws.

\subsubsection{Open-Ended}

The traditional experimental teaching focuses on the verification of the learned knowledge. According to the experimental steps and methods specified in the experimental instruction, the theoretical knowledge is verified, and then the experimental report is written according to the specified format. Under the provisions of this comparative dogma, most students can only be limited to the experimental operation process of book examples. Therefore, students will not be able to give full play to their innovative ability, and most of the experimental results are not ideal. Students' experimental process is more like the process of verifying theorems and laws according to experimental requirements. However, in the digital inquiry laboratory, the laboratory can design experimental projects for students to choose according to its own characteristics. The experimental projects can be divided into three types: must do, limited choice and arbitrary choice. In addition to doing experiments, students can classify and choose according to their own needs and hobbies. In this way, students' autonomy will be greatly enhanced. Students can also conduct in-depth exploration on the basis of completing their own studies. At the same time, because the efficiency of experimental data recording and collection process is greatly improved, students can also use their spare time to design experiments by themselves, carry out independent exploration under the guidance of teachers, and improve their self-learning ability, which is conducive to the development of students' personality and innovation ability.

\subsubsection{Integration}

In today's society with developed information technology, students' learning process is often disturbed by many external factors. Whether they have complete information processing ability is particularly critical. In the digital inquiry laboratory, students can more efficiently grasp the information processing link in the experimental process based on the real experiment and through the support of sensors, collectors and corresponding software, so as to effectively realize the integration of information technology and discipline. During the experiment, it will effectively prevent students from being helpless in the face of a large amount of data. At the same time, the effective software system equipped by the digital inquiry laboratory is effectively combined with the course experiment. At the 
beginning of the experiment, the experimental objectives and variables to be recorded in the experimental process are defined. Therefore, the experiment in the digital inquiry laboratory can timely avoid the dilemma that the experiment cannot be carried out due to the lack of teacher guidance.

\section{The Role of Digital Inquiry Laboratory in Teaching}

\subsection{Effectively Improve Classroom Efficiency}

In the traditional experimental class, due to the influence of traditional teaching ideas and the limitations of various factors. The teaching process of all experimental courses is basically the same. Before class, students preview according to the experimental teaching materials and write a preview report. During class, teachers explain the experimental principle, experimental steps and experimental data processing methods according to the experimental instruments. Students complete the experiment according to the teacher's explanation and go back to complete the experimental report. The direct result of this teaching method is that students have no enthusiasm for the experimental course, and most of them choose to complete the basic course tasks according to the experimental requirements, which suppresses students' enthusiasm and initiative. The experimental class is mainly based on Teachers' demonstration and students' step-by-step. Most of the time is spent on the collection and sorting of experimental data, and the teaching efficiency is low.

However, in the digital inquiry laboratory, the intervention of sensors and collectors makes the students' experimental process more oriented, the experimental progress more smoothly, and greatly improves the classroom efficiency.

\subsection{Solve the Problem of Poor Effect of Traditional Experiment}

In the traditional laboratory environment, due to a series of problems such as large error of manual data acquisition, slow data acquisition and inconvenient reading, the experimental effect is poor and the error is large, which is not conducive to the development of physics. (Research on the application of digital inquiry laboratory in physics teaching) especially in some experiments in which the experimental data change rapidly in a short time and are difficult to be accurately recorded, the students in the traditional laboratory will not be able to obtain the experimental results accurately and conveniently, thus affecting the experimental results.

By using sensors, the data transmitted to the data collector must be true and accurate. Therefore, the problem of repeated experiments caused by students' inability to accurately collect data, which directly affects the experimental effect, is avoided to a great extent.

\subsection{Stimulate Students' Initiative to Explore}

The openness of digital inquiry laboratory gives students more freedom in the process of experiment. Both the openness of time, space and course content ena- 
ble students to participate more in the experiment. The accurate and fast experimental data acquisition method avoids the possibility of large error of experimental results caused by equipment error and other reasons. In the digital inquiry laboratory, students can improve the experimental efficiency and have more free time to explore the problems they are interested in. At the same time, with the knowledge of teachers, they can deeply explore the experimental content they want to explore. Compared with traditional laboratories, these superior conditions enable students to obtain more sense of achievement in experimental activities and increase their enthusiasm for active exploration.

\section{Conclusion}

With the continuous advancement of educational reform, digital laboratory will be the development trend of experimental teaching in the future. More and more digital laboratories will be built, which requires primary and secondary school teachers to master this new experimental teaching means in the future. However, the application of digital inquiry laboratory is not impeccable. After all, information teaching is still in the initial stage, such as technical failure, unskilled equipment operation, poor application and many other problems. However, the integration of informatization and subject teaching is the inevitable trend of development. Only by constantly exploring and improving can we obtain better educational and teaching benefits.

\section{Funding}

Planning subject for Zhejiang Community Education (SQ21z02); Special project of lifelong education of Zhejiang Open University in 2021 (ZSJY202115).

\section{Conflicts of Interest}

The author declares no conflicts of interest regarding the publication of this paper.

\section{References}

Hao, X. X., Geng, S. J., Li, Y., Duan, X. J., Wang, J. F., \& Ren, M. J. (2015). Application Evaluation of Digital Microscope Network Interactive Laboratory in Histology Experiment Teaching-Taking Inner Mongolia Medical University as an Example. Heilongjiang Animal Husbandry and Veterinary, No. 24, 211-213.

Jiang, Y. Z., Ni, D. W., \& Liu, C. H. (2021). Methods and Paths of Laboratory Digital Transformation. Quality and Certification, No. 6, 53-55.

Li, F. (2019). Research on the Application of Digital Inquiry Laboratory in Physics Experiment Teaching in Senior High School. Contemporary Educational Practice and Teaching Research, No. 8, 18-19.

Liu, X. P., Wang, Y. J., \& Yin, H. (2018). Research on Digital and Intelligent Construction of Open Laboratory. Experimental Technology and Management, No. 10, 214-217.

Ma, S. P., \& Li, Y. (2012). Application Research of Digital Laboratory. Chinese Modern Educational Equipment, No. 14, 46-47. 
Pan, D. B., Luo, Z. Y., \& He, S. H. (2020). Exploration on Digital Ecological Management of Laboratory Personnel Ability. China Pharmaceutical, No. 5, 570-573.

Qian, Y. Y., \& Deng, F. (2006). The Construction of Digital Chemistry Inquiry Laboratory and the Cultivation of Students' Inquiry Ability. Audio Visual Education in China, No. $11,49-52$.

Troutman, V. A., \& Grimm, M. J. (2020). Interactive Digital Experience as an Alternative Laboratory (IDEAL): Creative Investigation of Forensic Biomechanics. Journal of Applied Biomechanics, 37, 163-170. https://doi.org/10.1123/jab.2020-0171

Viitaharju, P., Yliniemi, K., Nieminen, M., \& Karttunen, A. J. (2021). Learning Experiences from Digital Laboratory Safety Training. Education for Chemical Engineers, 34, 87-93. https://doi.org/10.1016/j.ece.2020.11.009

Wang, B. G. (2020). Analysis on the Training Mode of Applied Talents in Digital Garment Laboratory. Industry and Technology Forum, No. 20, 170-171.

Wang, H., Dai, F. H., Li, W. B., Li, R. F., \& He, Z. H. (2020). Application of Big Data in Digital Laboratory. Science, Technology and Innovation, No. 23, 54-55. 